\title{
Optimal Unimodular Sequences Design Method for Active Sensing Systems
}

\author{
Ze Li $(\mathbb{D}$, Ping Li, Xinhong Hao, and Xiaopeng Yan \\ Science and Technology on Electromechanical Dynamic Control Laboratory, Beijing Institute of Technology, Beijing 100081, China \\ Correspondence should be addressed to Xiaopeng Yan; yanxiaopeng@bit.edu.cn
}

Received 7 December 2017; Accepted 22 March 2018; Published 10 May 2018

Academic Editor: Kishin Sadarangani

Copyright (C) $2018 \mathrm{Ze} \mathrm{Li} \mathrm{et} \mathrm{al.} \mathrm{This} \mathrm{is} \mathrm{an} \mathrm{open} \mathrm{access} \mathrm{article} \mathrm{distributed} \mathrm{under} \mathrm{the} \mathrm{Creative} \mathrm{Commons} \mathrm{Attribution} \mathrm{License,} \mathrm{which}$ permits unrestricted use, distribution, and reproduction in any medium, provided the original work is properly cited.

\begin{abstract}
In active sensing systems, unimodular sequences with low autocorrelation sidelobes are widely adopted as modulation sequences to improve the distance resolution and antijamming performance. In this paper, in order to meet the requirements of specific practical engineering applications such as suppressing certain correlation coefficients and finite phase, we propose a new algorithm to design both continuous phase and finite phase unimodular sequences with a low periodic weighted integrated sidelobe level (WISL). With the help of the transformation matrix, such an algorithm decomposes the $N$-dimensional optimization problem into $N$ one-dimensional optimization problems and then uses the iterative method to search the optimal solutions of the $N$ onedimensional optimization problems directly. Numerical experiments demonstrate the effectiveness and the convergence property of the proposed algorithm.
\end{abstract}

\section{Introduction}

Since random sequences with low autocorrelation sidelobes can greatly improve the distance resolution of the detection signal, they are widely adopted as modulation sequences in active sensing systems, like radar, communication, proximity fuze, and so on [1-4]. Moreover, due to the limitations of hardware conditions (such as digital-to-analog converters, power amplifiers), it is usually more desirable to transmit unimodular sequences (i.e., constant modulus) to make full utilization of the transmitter's power $[5,6]$. So far, the most common modulation sequences applied in practical engineering are the pseudorandom code binary sequences, like $\mathrm{m}$ sequence, Gold sequence, chaotic sequence, and so on. Based on a binary phase shift keying modulation scheme, the pseudorandom code sequence usually corresponds to simple system hardware, but it is easy to crack. For instance, the $m$ sequence can be easily cracked by estimating its primitive polynomial with the help of third-order correlation function [7]. Therefore, in order to improve both the distance resolution and antijamming performance of active sensing systems, it is necessary to carry on waveform design to obtain unimodular sequences with good autocorrelation property.
According to the definition, the autocorrelation can be distinguished from periodic autocorrelation and aperiodic autocorrelation. Let $\{x(n)\}_{n=1}^{N}$ denote the unimodular sequence to be designed. The periodic $(r(k))$ and aperiodic $(\widehat{r}(k))$ autocorrelations of $\{x(n)\}_{n=1}^{N}$ are defined as

$$
\begin{array}{r}
r(k)=\sum_{n=1}^{N} x(n) x^{*}(n+k)(\bmod N)=r^{*}(-k), \\
k=0, \ldots, N-1, \\
\widehat{r}(k)=\sum_{n=1}^{N-k} x(n) x^{*}(n+k)=\widehat{r}^{*}(-k), \\
k=0, \ldots, N-1 .
\end{array}
$$

Then the integrated sidelobe level (ISL) which can be used to measure the quality of the autocorrelation property of a sequence is defined as

$$
\mathrm{ISL}=\sum_{k=-N+1, k \neq 0}^{N}|r(k)|^{2}=2 \sum_{k=1}^{N-1}|r(k)|^{2} .
$$


The smaller the ISL of a sequence, the better the autocorrelation property of this sequence. The above formula shows the definition of ISL metric for periodic autocorrelations, and the ISL metric for the aperiodic autocorrelations can be defined similarly. It is worth noting that the ISL metric is equivalent to another very important measure, the merit factor (MF), and the relationship between the two is as follows:

$$
\mathrm{MF}=\frac{\left|r_{0}\right|^{2}}{2 \sum_{k=1}^{N-1}|r(k)|^{2}}=\frac{N^{2}}{2 \mathrm{ISL}} .
$$

Correspondingly, the higher the MF of a sequence, the better the autocorrelation property of this sequence.

The simple analysis described above indicates that to improve the autocorrelation property of a sequence it is necessary to optimize each code of the sequence, which means that the optimization problem is essentially an $N$ dimensional optimization problem, and its optimal solution cannot be obtained directly. In order to solve this problem, the majority of scholars have proposed a number of algorithms to obtain the optimal solution [8-22]. Since the $N$ dimensional optimization problem may have multiple local optimal solutions, the simulated annealing [8] and stochastic optimization methods $[9,10]$ were suggested to obtain the global optimal solution at the early stage. However, as the length of the sequence $(N)$ grows, the computational burdens of these methods increase exponentially. Therefore, in order to design a sequence with a long length, both the effectiveness and convergence property of the algorithm need to be taken into account. Recently, two groups of typical algorithms [1118 ] with superiorities in the above two aspects have attracted extensive attention from scholars. The work in [11] proposed an iterative algorithm called CAN (Cyclic Algorithm-New) to design a unimodular sequence with low aperiodic ISL. The algorithm deduces the relationship between the aperiodic ISL and the Fourier spectrum of the sequence, which makes it possible to significantly improve the computational efficiency of such an algorithm by implementing fast Fourier transform (FFT) operations in the process of seeking the optimal solution. In addition, differently modified or extended versions of such algorithms have been applied to generate unimodular sequences with low periodic ISL (PeCAN) [12], unimodular sequences with low aperiodic weighted ISL (WeCAN) [11], and complementary sets of sequences (CSS) with both good autocorrelation and cross-correlation properties for MIMO radar systems (CANARY) [13-15]. The work in [16] proposed another iterative algorithm called MISL (monotonic minimizer for ISL). Based on the general majorizationminimization scheme, the MISL algorithm constructs the upper limit function of objective function twice so as to transform the complex ISL optimization problem into a simple optimization problem. Meanwhile, it is pointed out in [16] that by replacing the corresponding FFT matrix the MISL algorithm can also be applied to deal with the periodic ISL optimization problem. Subsequently, the authors improved the MISL algorithm (MWISL) in [17] to deal with the aperiodic weighted ISL optimization problem and provided several extended versions of the MISL algorithm in [18] for the design of CSS with both good aperiodic autocorrelation and cross-correlation properties. In addition to the two groups of typical algorithms mentioned above, some novel algorithms were proposed in the same period. For instance, [19] introduces a computational framework based on an iterative twisted approximation (ITROX) which can deal with various sequence design problems. However, ITROX needs to do eigenvalue decomposition at each iteration, which limits the application of such algorithms in the design of long sequences. The work in [20] proposed an iteration direct search optimization algorithm to design a constant modulus sequence set with low aperiodic WISL. Such an algorithm solves the optimization problem by optimizing each code of the sequence one by one directly, which greatly reduces the number of iterations and ensures the convergence property. Besides the correlation properties, some of the latest algorithms have also begun to take into account the other properties of the sequence. The work in [21] proposed a gradient-based algorithm named gradient-weighted correlation-SFW (Gra-WeCorr-SFW) to design unimodular sequences sets with both good aperiodic correlation and stopband properties. Furthermore, [22] proposed the design of unimodular sequences whose aperiodic autocorrelation and aperiodic complementary autocorrelation vanish for a given set of lags.

Most of the above literature is focused on the aperiodic correlation property of sequences with continuous phase, and the sequence with low aperiodic correlation is mainly used in the active sensing system with intrapulse coded modulation such as the pulse compression radar [23]. However, some active sensing systems such as proximity fuzes [24] and multiple modulation detectors [25] adopt interpulse coded modulation and therefore consider the periodic correlation property of modulation sequence rather than the aperiodic correlation property. In addition, for some practical engineering applications, it is not yet possible to achieve continuous phase modulation, mainly in regard to the hardware condition of detectors, especially miniaturization detectors, and the Doppler tolerance deterioration caused by continuous phase modulation. Consequently, for cases of specific applications, finite phase unimodular sequence with good periodic correlation property is demanded.

However, to our knowledge, less attention is paid to the research on the periodic correlation property of sequences with finite phase. In this paper, considering cases of specific applications, we propose a new algorithm called the Periodic correlation Weighted Cyclic Iteration Algorithm (PWCIA), which can be used to generate both continuous phase and finite phase unimodular sequences with low periodic weighted ISL. With the help of the transformation matrix, the PWCIA decomposes the $N$-dimensional sequence optimization problem into $N$ one-dimensional sequence optimization problems and uses the iterative method to search the optimal solutions of the $N$ one-dimensional optimization problems directly. The convergence of the algorithm is analyzed theoretically, and the FFT operations are implemented to ensure the actual convergence speed of the algorithm. Numerical experiments indicate that compared with other algorithms the proposed PWCIA has the advantages of both periodic autocorrelation property and convergence property. 
The rest of the paper is organized as follows. In Section 2, we formulate the optimization problem and review the existing algorithm. In Section 3, we deduce and present the PWCIA. In Section 4, we evaluate the performance of the PWCIA via a number of numerical experiments. Finally, in Section 5, we provide the conclusions.

Notation. Boldface uppercase letters denote matrices, and boldface lowercase letters denote column vectors. $\operatorname{Re}(\cdot)$ and $\operatorname{Im}(\cdot)$ denote the real and imaginary part. $(\cdot)^{*},(\cdot)^{T}$ and $(\cdot)^{H}$ denote complex conjugate, transpose, and conjugate transpose, respectively. $\arg (\cdot)$ denotes the phase of a complex number. $a(n)$ denotes the $n$th element of the vector a. $\|\cdot\|_{2}$ and $|\cdot|$ denote the Frobenius norm and the modulus.

\section{Problem Formulation and Existing Methods}

In order to obtain good distance resolution and antijamming performance, it needs to improve the processing gain of active sensing systems. For active sensing systems which adopt interpulse coded modulation, such a goal can be achieved by designing unimodular sequences with low periodic autocorrelation sidelobes as modulation sequences. Hence, the issue of concern in this paper is actually to design the unimodular sequence with good periodic autocorrelation property.

2.1. Problem Formulation. In the introduction, we let $\{x(n)\}_{n=1}^{N}$ denote the unimodular sequence to be designed (i.e., $|x(n)|=1, n=1,2, \ldots, N)$; then any code $x(n)$ in the sequence can be expressed as

$$
x(n)=\exp \{j \phi(n)\},
$$

where $\phi(n)$ denotes the phase of corresponding code $x(n)$. We define $\Phi=[\phi(1), \phi(2), \ldots, \phi(N)]^{T}$ as the phase vector of the unimodular sequence. For the unimodular sequence with arbitrary continuous phase, the value of $\phi(n)$ can be any real number in the interval, that is, $\phi(n) \in[0,2 \pi)$, while for the unimodular sequence with finite phase the value of $\phi(n)$ is discrete and its range is $\phi(n) \in[0,2 \pi / q, 4 \pi / q, \ldots,(2 \pi / q)(q-$ $1)]$, where $q$ denotes the number of finite phases. Thus, according to formula (1), the periodic autocorrelation $r(k)$ of sequence $\mathbf{x}$ can be expressed as

$$
\begin{aligned}
r(k) & =r^{*}(-k)=\sum_{n=1}^{N} x(n) x^{*}(n+k)(\bmod N) \\
& =\sum_{n=1}^{N} \exp \{j[\phi(n)-\phi(n+k)(\bmod N)]\} .
\end{aligned}
$$

Combining (3) and (6), the weighted integrated sidelobe level (WISL) can be defined as

$$
\mathrm{WISL}=\sum_{k=-N+1, k \neq 0}^{N} w_{k}|r(k)|^{2}=2 \sum_{k=1}^{N-1} w_{k}|r(k)|^{2},
$$

where $w_{k} \geq 0, k=1, \ldots, N-1$ denotes the weight of autocorrelation coefficient corresponding to different lag time $k$. By setting the value of $\left\{w_{k}\right\}_{k=1}^{N-1}$, we can arbitrarily adjust the side lobe level at any lags to meet different actual requirements (such as suppressing the interference signal within a certain distance). In particular, ISL metric can be regarded as a special case of WISL metric, by simply taking $w_{k}=1, k=1, \ldots, N-1$.

The problem of interest in this paper is the following WISL minimization problem:

$$
\begin{array}{ll}
\underset{x(n)}{\operatorname{Minimize}} & \text { WISL } \\
\text { Subject to } & |x(n)|=1, \quad n=1,2, \ldots, N .
\end{array}
$$

So far, the research work on unimodular sequences with a good aperiodic autocorrelation property has been carried out a lot, but the typical algorithm for designing unimodular sequences with a good periodic autocorrelation property is just PeCAN (Periodic correlation Cyclic Algorithm-New), which we will briefly review before we introduce the proposed algorithm in this paper.

2.2. Existing Methods. The CAN (Cyclic Algorithm-New) and WeCAN (Weighted Cyclic Algorithm-New) were proposed in [11] for separately designing unimodular sequences with low ISL and low WISL. The above two algorithms concern the aperiodic correlation property of the sequence, but in this paper, according to the actual engineering requirements, we consider the periodic correlation property of the sequence. It can be seen from (1) and (2) that the periodic autocorrelation coefficient $r(k)$ and the aperiodic autocorrelation coefficient $\widehat{r}(k)$ greatly differ on the definition, and thus CAN and WeCAN are not suitable for the periodic correlation case.

In the follow-up research work, the authors proposed a PeCAN similar to CAN specifically to design a unimodular sequence with low ISL of periodic correlation [12]. Instead of minimizing the ISL metric directly, the authors proposed solving the following simpler problem:

$$
\begin{array}{ll}
\underset{x(n), \psi(p)}{\operatorname{Minimize}} & \sum_{m=1}^{N}\left|f(p)-\sqrt{N} e^{j \psi(p)}\right|^{2}, \\
\text { subject to } & |x(n)|=1, \quad n=1, \ldots, N,
\end{array}
$$

where

$$
f(p)=\sum_{n=1}^{N} x(n) e^{(-j 2 \pi / N) p n}, \quad p=1, \ldots, N
$$

denotes the finite Fourier transform of the sequence $\{x(n)\}_{n=1}^{N}$, and $\{\psi(p)\}$ are auxiliary variables. The authors had proved that problem (9) is "almost equivalent" to the original problem (8) with $w_{k}=1, k=1, \ldots, N-1$.

Let $\mathbf{F}$ denote the $N \times N$ FFT matrix. The objective function of problem (9) can be rewritten in the following more compact form:

$$
\|\mathbf{F x}-\sqrt{N} \mathbf{v}\|^{2}
$$

where $\mathbf{v}=\left[e^{j \psi(1)}, \ldots, e^{j \psi(N)}\right]^{T}, \mathbf{x}=[x(1), \ldots, x(N)]^{T}$. The PeCAN then minimizes (11) by alternating between $\mathbf{x}$ and 
(1) Set the $\{x(n)\}_{n=1}^{N}$ to an initial values.

(2) Compute $[f(1), \ldots, f(N)]^{T}=\mathbf{F x} ; v(p)=e^{j \arg (f(p))}, p=1, \ldots, N$.

(3) Compute $[z(1), \ldots, z(N)]^{T}=\mathbf{F}^{H} \mathbf{v} ; x(n)=e^{j \arg (z(n))}, n=1, \ldots, N$.

(4) Iterate steps (2) and (3) until convergence.

Algorithm 1: The PeCAN proposed in [12].

v. For given $\{x(n)\}_{n=1}^{N}$, compute the FFT of $\{x(n)\}_{n=1}^{N}$, that is, $\{f(p)\}_{p=1}^{N}$, and the minimization of (11) with respect to $\{\psi(p)\}_{p=1}^{N}$ yields

$$
\psi(p)=\arg (f(p)), \quad p=1, \ldots, N .
$$

Similarly, for given $\{\psi(p)\}_{p=1}^{N}$, compute the IFFT of $\mathbf{v}$, let us say $\{z(n)\}_{n=1}^{N}$, and the minimization of (11) with respect to $\{x(n)\}_{n=1}^{N}$ is given by

$$
x(n)=e^{j \arg (z(n))}, \quad n=1, \ldots, N .
$$

The PeCAN for minimizing of the periodic correlation ISL metric in (9) is summarized as shown in Algorithm 1.

It was shown numerically in [12] that PeCAN can generate almost perfect (with zero ISL) sequences from random initializations. However, the authors have not proposed an algorithm to design the unimodular sequences with low WISL of periodic autocorrelations. In addition, PeCAN is originally proposed to design sequences with continuous phase, while according to the actual engineering requirements we need to design sequences with finite phase in this paper. Therefore, the PeCAN does not have the full ability to solve the problem raised in this paper, and we will propose the algorithm to solve problem (8) for both continuous phase case and finite phase case.

\section{PWCIA (Periodic Correlation Weighted Cyclic Iteration Algorithm)}

To design finite phase unimodular sequences with a low periodic weighted ISL metric which is suitable for the cases of specific applications mentioned before, we propose a new cyclic algorithm in this section. It is worth noting that although we are motivated by the algorithm in [20], we deal with problem (8) for the periodic correlation case while the former is used to deal with the aperiodic correlation case.

3.1. PWCIA Procedure. Let $\Delta \phi$ be an arbitrary phase increment of $n$th element of the sequence $\{x(n)\}_{n=1}^{N}$, and define a new unimodular sequence $\widetilde{\mathbf{x}}$ as

$$
\begin{aligned}
\tilde{x}(k) & = \begin{cases}x(k) & k \neq n \\
x(k) \exp \{j \Delta \phi\} & k=n\end{cases} \\
& = \begin{cases}\exp \{j \phi(k)\} & k \neq n \\
\exp \{j \phi(k)+j \Delta \phi\} & k=n .\end{cases}
\end{aligned}
$$
by

Let $\mathbf{A}$ be the autocorrelation matrix of $\mathbf{x}$, which is given

$$
\begin{aligned}
\mathbf{A} & =\mathbf{x x}^{H} \\
& =\left[\begin{array}{cccc}
x(1) x^{*}(1) & x(1) x^{*}(2) & \cdots & x(1) x^{*}(N) \\
x(2) x^{*}(1) & x(2) x^{*}(2) & \cdots & x(2) x^{*}(N) \\
\vdots & \vdots & \ddots & \vdots \\
x(N) x^{*}(1) & x(N) x^{*}(2) & \cdots & x(N) x^{*}(N)
\end{array}\right] .
\end{aligned}
$$

With the help of autocorrelation matrix $\mathbf{A}$, the periodic autocorrelation function $r(k)$ in (6) can be rewritten as

$$
\begin{aligned}
& r(k)= r^{*}(-k) \\
&= \sum_{j=1}^{N-k} \mathbf{A}(j+k, j)+\sum_{j=1}^{k} \mathbf{A}(j, j+N-k), \\
& \quad k=0, \ldots, N-1 .
\end{aligned}
$$

Consider the new autocorrelation matrix $\widetilde{\mathbf{A}}=\widetilde{\mathbf{x}} \widetilde{\mathbf{x}}^{H}$; compared with $\mathbf{A}$, only the elements of the $n$th row and the $n$th column are changed. Furthermore, the exchanged vectors $\mathbf{q}_{r, n}$ and $\mathbf{q}_{c, n}$ can be defined as

$$
\begin{aligned}
\mathbf{q}_{r, n} & =x(n)\left[x^{*}(n-1), x^{*}(n-2), \ldots, x^{*}(1), x^{*}(N),\right. \\
x^{*} & \left.(N-1), \ldots, x^{*}(n+1)\right]^{T}, \\
\mathbf{q}_{c, n} & =x^{*}(n)[x(n+1), x(n+2), \ldots, x(N), x(1), x(2), \\
& \ldots, x(n-1)]^{T} .
\end{aligned}
$$

It is worth noting that we proposed the autocorrelation matrix A to show the relationship between $r(k)$ and $\widetilde{r}_{n}(k)$, but in practice the autocorrelation function $r(k)$ can be efficiently obtained via FFT operation. Let $\mathbf{f}=\mathbf{F x}$ denote the FFT of the original sequence $\mathbf{x}$. It is well known that according to the periodic correlation theorem the periodic autocorrelation function $\mathbf{r}$ can be written as

$$
\mathbf{r}=\mathbf{F}^{H}|\mathbf{f}|^{2}
$$

Owing to the FFT operation, the calculation speed of this algorithm is greatly improved. After the autocorrelation function $\mathbf{r}$ and the exchanged vectors are obtained, the 
autocorrelation function for the new sequence can be expressed as

$$
\begin{array}{r}
\tilde{r}_{n}(k)=r(k)-\left(\mathbf{q}_{r, n}(k)+\mathbf{q}_{c, n}(k)\right) \\
+\left(\mathbf{q}_{r, n}(k) \exp (j \Delta \phi)+\mathbf{q}_{c, n}(k) \exp (-j \Delta \phi)\right), \\
k=1, \ldots, N-1 .
\end{array}
$$

Let

$$
g_{n}(k)=r(k)-\mathbf{q}_{r, n}(k)-\mathbf{q}_{c, n}(k) .
$$

Then $\left|\widetilde{r}_{n}(k)\right|^{2}$ can be described as

$$
\left|\widetilde{r}_{n}(k)\right|^{2}=\left\{\operatorname{Re}\left\{\widetilde{r}_{n}(k)\right\}\right\}^{2}+\left\{\operatorname{Im}\left\{\widetilde{r}_{n}(k)\right\}\right\}^{2},
$$

where

$$
\begin{aligned}
\operatorname{Re}\left\{\widetilde{r}_{n}(k)\right\}= & \operatorname{Re}\left\{g_{n}(k)\right\}+\operatorname{Re}\left\{\mathbf{q}_{r, n}(k)\right\} \cos \Delta \phi \\
& -\operatorname{Im}\left\{\mathbf{q}_{r, n}(k)\right\} \sin \Delta \phi \\
& +\operatorname{Re}\left\{\mathbf{q}_{\mathrm{c}, n}(k)\right\} \cos \Delta \phi \\
& +\operatorname{Im}\left\{\mathbf{q}_{\mathrm{c}, n}(k)\right\} \sin \Delta \phi ; \\
\operatorname{Im}\left\{\widetilde{r}_{n}(k)\right\}= & \operatorname{Im}\left\{g_{n}(k)\right\}+\operatorname{Re}\left\{\mathbf{q}_{r, n}(k)\right\} \sin \Delta \phi \\
& +\operatorname{Im}\left\{\mathbf{q}_{r, n}(k)\right\} \cos \Delta \phi \\
& -\operatorname{Re}\left\{\mathbf{q}_{\mathrm{c}, n}(k)\right\} \sin \Delta \phi \\
& +\operatorname{Im}\left\{\mathbf{q}_{c, n}(k)\right\} \cos \Delta \phi .
\end{aligned}
$$

Then (21) can be simplified as

$$
\begin{aligned}
\left|\widetilde{r}_{n}(k)\right|^{2}= & a_{0, k}+a_{1, k} \cos \Delta \phi+a_{2, k} \sin \Delta \phi \\
& +a_{3, k} \cos 2 \Delta \phi+a_{4, k} \sin 2 \Delta \phi, \\
& \quad k=1, \ldots, N-1,
\end{aligned}
$$

where

$$
\begin{aligned}
a_{0, k}= & |g(k)|^{2}+\left|\mathbf{q}_{r, n}(k)\right|^{2}+\left|\mathbf{q}_{c, n}(k)\right|^{2} ; \\
a_{1, k}= & 2 \operatorname{Re}\left\{g_{n}(k)\right\}\left(\operatorname{Re}\left\{\mathbf{q}_{r, n}(k)\right\}+\operatorname{Re}\left\{\mathbf{q}_{c, n}(k)\right\}\right) \\
& +2 \operatorname{Im}\left\{g_{n}(k)\right\}\left(\operatorname{Im}\left\{\mathbf{q}_{r, n}(k)\right\}+\operatorname{Im}\left\{\mathbf{q}_{c, n}(k)\right\}\right) ; \\
a_{2, k}= & 2 \operatorname{Im}\left\{g_{n}(k)\right\}\left(\operatorname{Re}\left\{\mathbf{q}_{r, n}(k)\right\}-\operatorname{Re}\left\{\mathbf{q}_{c, n}(k)\right\}\right) \\
& -2 \operatorname{Re}\left\{g_{n}(k)\right\}\left(\operatorname{Im}\left\{\mathbf{q}_{r, n}(k)\right\}-\operatorname{Im}\left\{\mathbf{q}_{c, n}(k)\right\}\right) ;
\end{aligned}
$$

$$
\begin{aligned}
a_{3, k}= & 2 \operatorname{Re}\left\{\mathbf{q}_{r, n}(k)\right\} \operatorname{Re}\left\{\mathbf{q}_{c, n}(k)\right\} \\
& +2 \operatorname{Im}\left\{\mathbf{q}_{r, n}(k)\right\} \operatorname{Im}\left\{\mathbf{q}_{c, n}(k)\right\} ; \\
a_{4, k}= & 2 \operatorname{Re}\left\{\mathbf{q}_{r, n}(k)\right\} \operatorname{Im}\left\{\mathbf{q}_{c, n}(k)\right\} \\
& -2 \operatorname{Re}\left\{\mathbf{q}_{c, n}(k)\right\} \operatorname{Im}\left\{\mathbf{q}_{r, n}(k)\right\} .
\end{aligned}
$$

Substituting (23) into (7), then the weighted ISL of the new sequence can be computed as

$$
\begin{aligned}
& \widetilde{\mathrm{WISL}}_{n}(\Delta \phi)=2 \sum_{k=1}^{N-1} w_{k}\left|\widetilde{r}_{n}(k)\right|^{2}=2\left(\alpha_{n, 0}\right. \\
& +\alpha_{n, 1} \cos \Delta \phi+\alpha_{n, 2} \sin \Delta \phi+\alpha_{n, 3} \cos 2 \Delta \phi \\
& \left.+\alpha_{n, 4} \sin 2 \Delta \phi\right)
\end{aligned}
$$

where $\alpha_{n, l}=\sum_{k=1}^{N-1} w_{k} a_{l, k}, l=0,1,2,3,4$. After we get (25), the original problem (8) is decomposed into $N$ onedimensional problems which can be described as

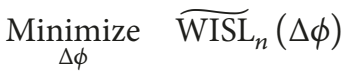

$$
\begin{aligned}
& \text { Subject to } \Delta \phi \in \Psi \text {. }
\end{aligned}
$$

Because of the difference in the range of phase increment $\Delta \phi$, there will be two different kinds of solutions to problem (26).

For the continuous phase case, $\Psi=[-\pi, \pi]$, and the optimal solution $\Delta \phi^{*}$ can be computed by

$$
\Delta \phi^{*}=\arg \min _{\psi_{1}}\left\{\widetilde{\mathrm{WISL}}_{n}(\Delta \phi)\right\}
$$

where $\psi_{1} \subseteq \psi$, and $\psi_{1}$ denotes the set of all the real elments in $\left\{2 \arctan \left(x_{1}\right), 2 \arctan \left(x_{2}\right), 2 \arctan \left(x_{3}\right), 2 \arctan \left(x_{4}\right), \pi\right\}$. Here $x_{1}, x_{2}, x_{3}$, and $x_{4}$ are roots of equation $f_{n}(x)=0$, and $f_{n}(x)$ is defined in (29).

According to (25), the first partial derivatives of $\widetilde{\mathrm{WISL}}_{n}(\Delta \phi)$ about $\Delta \phi$ named $\widetilde{\mathrm{WISL}}_{n}{ }_{n}(\Delta \phi)$ can be described as

$$
\begin{aligned}
{\widetilde{\mathrm{WISL}_{n}}}_{n}^{\prime}(\Delta \phi)= & -2 \alpha_{n, 1} \sin \Delta \phi+2 \alpha_{n, 2} \cos \Delta \phi \\
& -4 \alpha_{n, 3} \sin 2 \Delta \phi+4 \alpha_{n, 4} \cos 2 \Delta \phi .
\end{aligned}
$$

Let $x=\tan (\Delta \phi / 2)$. With the help of the trigonometric universal formula, (28) turns into a 4th-order polynomial of the independent variable $x$, which can be described as

$$
\begin{aligned}
f_{n}(x) & =-2 \alpha_{n, 1} \frac{2 x}{1+x^{2}}+2 \alpha_{n, 2} \frac{1-x^{2}}{1+x^{2}}-4 \alpha_{n, 3} \frac{4 x\left(1-x^{2}\right)}{\left(1+x^{2}\right)^{2}}+4 \alpha_{n, 4} \frac{\left(1-x^{2}\right)^{2}-4 x^{2}}{\left(1+x^{2}\right)^{2}} \\
& =\frac{\left(4 \alpha_{n, 4}-2 \alpha_{n, 2}\right) x^{4}+\left(16 \alpha_{n, 3}-4 \alpha_{n, 1}\right) x^{3}-24 \alpha_{n, 4} x^{2}-\left(4 \alpha_{n, 1}+16 \alpha_{n, 3}\right) x+\left(2 \alpha_{n, 2}+4 \alpha_{n, 4}\right)}{\left(1+x^{2}\right)^{2}} .
\end{aligned}
$$


(1) Set the $\{x(n)\}_{n=1}^{N}$ to an initial value; and select the desired weights $\left\{w_{k} \geq 0\right\}_{k=1}^{N-1} ; i=0$;

(2) Set $n=1$;

(3) Compute $\mathbf{X}^{(i)}=\mathbf{F} \mathbf{x}^{(i)} ; \mathbf{r}^{(i)}=\mathbf{F}^{H}\left|\mathbf{X}^{(i)}\right|^{2}$;

(4) Compute the exchanged vectors $\mathbf{q}_{r, n}^{(i)}$ and $\mathbf{q}_{c, n}^{(i)}$ according to (17); Compute $a_{l, k}^{(i)}, l=0,1,2,3,4$ according to (23);

(5) Compute $\alpha_{n, l}^{(i)}=\mathbf{w}^{T} \mathbf{a}_{l}^{(i)}, l=0,1,2,3,4$; and get the target function $\widetilde{\mathrm{WISL}}_{n}^{(i)}(\Delta \phi)$;

(6) Obtain the optimal solution $\Delta \phi_{n}^{i}$ to the one-dimensional problem (26) according to (27) for continuous phase case or according to (30) for finite phase case.

(7) Update $x^{(i)}(n)=x^{(i-1)}(n) \exp \left\{j \Delta \phi_{n}^{i}\right\} ; n=n+1$;

(8) Judge $n$, if $n \leq N$, return to step (3); otherwise turn to step (9).

(9) If $\left\|\mathbf{x}^{(i)}-\mathbf{x}^{(i-1)}\right\|_{2}<\varepsilon$, where $\varepsilon$ is a predefined threshold to control convergence, output $\mathbf{x}^{*}=\mathbf{x}^{(i)}$; otherwise, $i=i+1$, return to step (2) until convergence.

Algorithm 2: PWCIA (Periodic correlation Weighted Cyclic Iteration Algorithm).

It is easy to see that the equation $f_{n}(x)=0$ has four roots in complex field, of which the real roots might be the local minimum point of $\widetilde{\mathrm{WISL}}_{n}(\Delta \phi)$.

For the finite phase case, $\Psi=[0,2 \pi / q, 4 \pi / q, \ldots$, $(2 \pi / q)(q-1)]$, where $q$ denotes the number of finite phases. The optimal solution $\Delta \phi^{*}(30)$ can be efficiently found by onedimensional exhaustive search procedure.

$$
\Delta \phi^{*}=\arg \min _{\Psi}\left\{\widetilde{\mathrm{WISL}}_{n}(\Delta \phi)\right\}
$$

After the optimal solution $\Delta \phi^{*}$ to problem (26) is obtained, the $n$th element of the sequence $\{x(n)\}_{n=1}^{N}$ can be updated as

$$
x(n)=x(n) \exp \left\{j \Delta \phi^{*}\right\} .
$$

According to the above derivation, we decompose the original $\mathrm{N}$-dimensional optimization problem (8) into $\mathrm{N}$ one-dimensional optimization problems (26) and give the optimal solution separately for the case of both continuous phase (27) and finite phase (30). Due to the intrinsic relationship of the elements in the sequence, we cannot obtain the optimal solution at one time, which requires us to solve the original problem by cyclic iteration. At each iteration, we sequentially optimize each code element, assuming the remaining $(N-1)$ code elements are fixed.

We call the cyclic iteration algorithm PWCIA (Periodic correlation Weighted Cyclic Iteration Algorithm), which is summarized as shown in Algorithm 2.

The algorithm PWCIA decomposes the $N$-dimensional optimization problem into $N$ one-dimensional optimization problems and searches the optimal solution directly at each iteration, which makes the convergence rate of this algorithm very fast.

3.2. Convergence Analysis. In this subsection, we briefly analyze the convergence of the proposed algorithm. Because $\Delta \phi_{n}^{i}$ is the optimal solution of problem (26) at ith iteration, we can guarantee the following inequality $\widetilde{\mathrm{WISL}}_{n}^{(i)}\left(\Delta \phi_{n}^{i}\right) \leq$ $\widetilde{\mathrm{WISL}}_{n}^{(i)}(\Delta \phi=0)=\widetilde{\mathrm{WISL}}_{n-1}^{(i)}\left(\Delta \phi_{n-1}^{i}\right)$. Let $\mathrm{WISL}_{n}^{(i)}$ denote objective function value for the optimization of the $n$th element of sequence at $i$ th iteration, and it is obvious that $\mathrm{WISL}_{n}^{(i)}=\widetilde{\mathrm{WISL}}_{n}^{(i)}\left(\Delta \phi_{n}^{i}\right)$. Based on the above discussion, we have

$$
\begin{aligned}
0 & <\operatorname{WISL}\left(\mathbf{x}^{(i)}\right)=\mathrm{WISL}_{N}^{(i)} \leq \mathrm{WISL}_{N-1}^{(i)} \leq \cdots \\
& \leq \mathrm{WISL}_{1}^{(i)} \leq \mathrm{WISL}\left(\mathbf{x}^{(i-1)}\right)
\end{aligned}
$$

where WISL $\left(\mathbf{x}^{(i)}\right)$ denotes the objective function value after all the elements of sequence $\mathbf{x}$ at $i$ th iteration are updated, and there is no doubt that WISL( $\mathbf{x})$ is greater than 0 . In summary, the objective function value decreases monotonically at each iteration and has a lower bound and therefore will eventually converge to a finite value.

\section{Numerical Experiments}

In this section, we evaluate the performance of the proposed PWCIA via numerical experiments for both the continuous and the finite phase cases. Since the existing algorithms for periodic autocorrelation can only be used to generate the unimodular sequence with low ISL metric, while the PWCIA can deal with the weighted ISL metric in addition to the ISL metric, we specially design experiments for the ISL metric in order to compare the performance of the proposed PWCIA with the existing algorithm in the first subsection. Then, in the second subsection, we design experiments to evaluate the performance of the PWCIA for WISL metric. All experiments were performed on a PC with a $2.7 \mathrm{GHz}$ Core i5-7200U CPU and 8 GB RAM.

4.1. ISL Metric. In this subsection, we compare the performance of the proposed PWCIA with the PeCAN. The Matlab code of the original PeCAN was downloaded from the website of the book [4]. Without the loss of generality, all the sequences in the simulation are initiated by the unit energy sequence with random phases.

4.1.1. Continuous Phase Scenario. We first compare the quality, measured by the merit factor (MF) defined in (4), of 


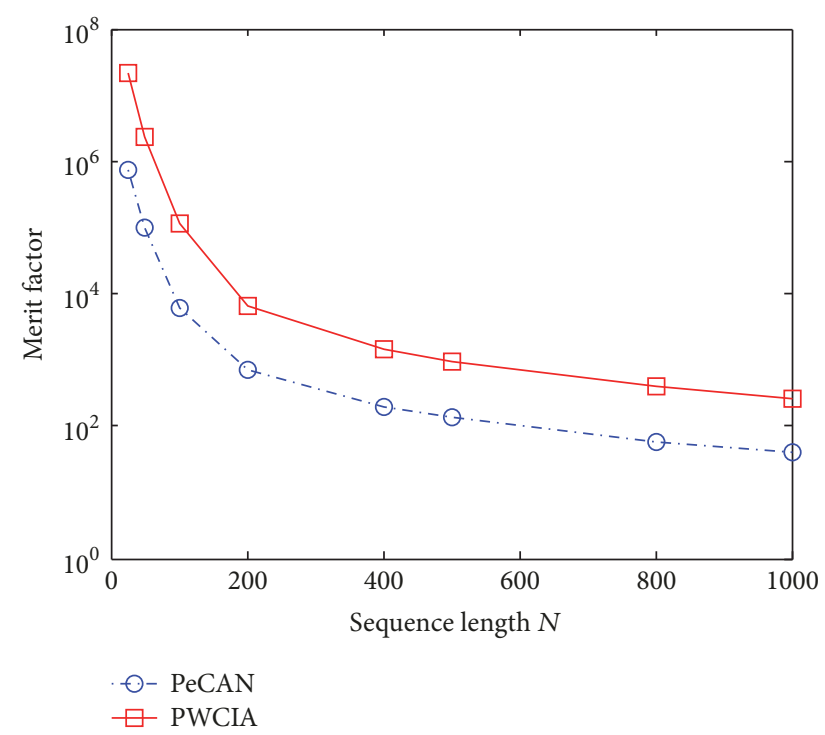

FIGURE 1: Merit factor (MF) versus sequence length. Each curve is averaged over 100 random trials.

the output sequences of different lengths generated by the PWCIA (with $w_{k}=1, k=1, \ldots, N-1$ ) and the PeCAN. In this experiment, for both algorithms, the stopping criterion is set to be $\left\|\mathbf{x}^{(i)}-\mathbf{x}^{(i-1)}\right\|_{2}<N \times 10^{-6}$. Both algorithms were repeated 100 times for each of the following lengths: $N=$ $25,50,100,200,400,500,800$, and 1000. Furthermore, for fair comparison, the PWCIA and the PeCAN are initialized by the same unit energy sequence with random phases at each time. The average merit factor of the output sequences generated by two different algorithms is shown in Figure 1 using a semilog scale. From Figure 1, we can see that the proposed PWCIA can generate sequences with a consistently larger MF than the PeCAN for all lengths under the same condition. Furthermore, because the stopping criterion relaxes as the sequence length grows, the merit factor of generated sequences with short length is larger than the long ones for both two algorithms. The periodic correlation levels of two example sequences with length $N=100$ and $N=500$ generated by the two algorithms are shown in Figure 2, where the periodic correlation level is defined as

$$
\begin{aligned}
\text { periodic correlation level }=20 \log _{10}\left|\frac{r(k)}{r_{0}}\right|, \\
k=1-N, \ldots, N-1 .
\end{aligned}
$$

It is worth noting that both algorithms are sensitive to the initial sequences. In this experiment, for the sequence lengths of $N=25,50$, and 100, the case in which the MF of the PeCAN sequence is larger than the PWCIA sequence happens occasionally among the 100 random trials. However, for other sequence lengths, the MF of PWCIA sequence is always larger than the PeCAN sequence which is initialized by the same random sequence.

In the following, we perform an experiment to show the convergence properties of the two algorithms. Particularly,

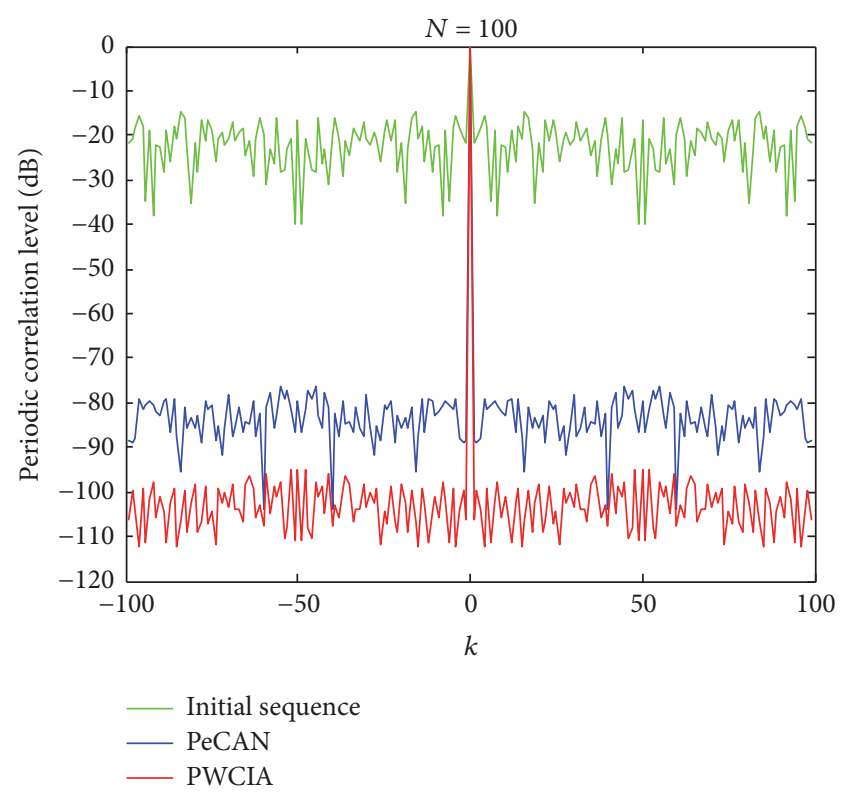

(a)

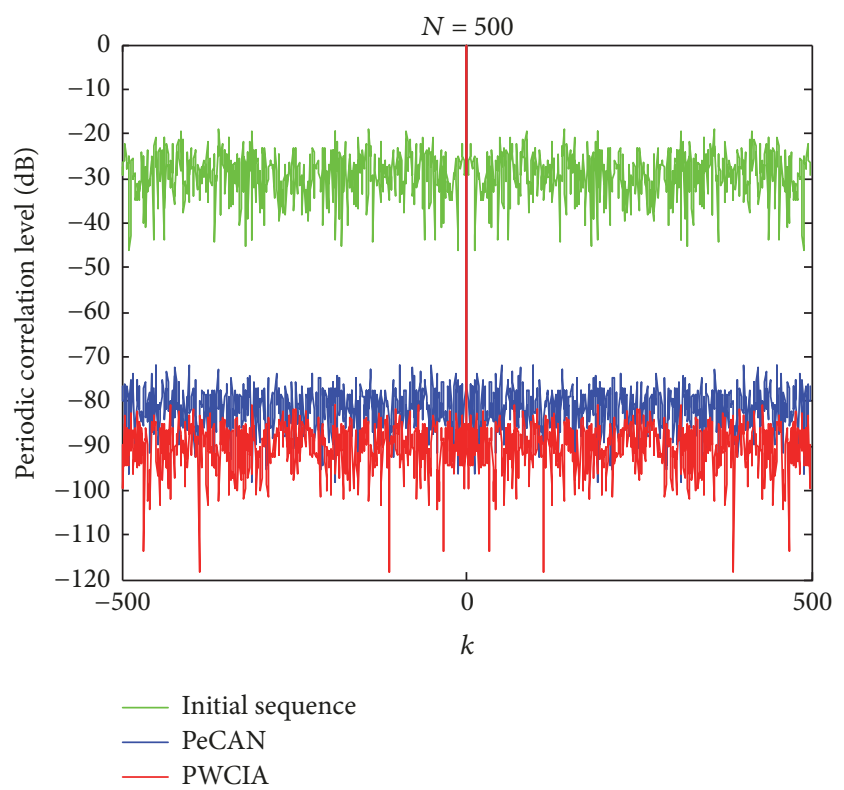

(b)

Figure 2: Periodic correlation levels of initial sequence and sequences generated by PeCAN and PWCIA of lengths $N=100$ and $N=500$. (a) $N=100$; (b) $N=500$.

in this experiment, we consider the length of the designed sequence $N=100$ and for both algorithms the stopping criterion is set to be ISL $<10^{-10}$. The ISL versus the iteration number and the corresponding computational time are shown in Figure 3. From Figures 3(a) and 3(b), we can see that both the ISL of the PWCIA and the PeCAN decrease monotonically as the iteration number increases, but the converged speeds are different. Compared with the PeCAN, the PWCIA needs much more time to conduct an iteration, but the ISL of the PWCIA decreases faster 


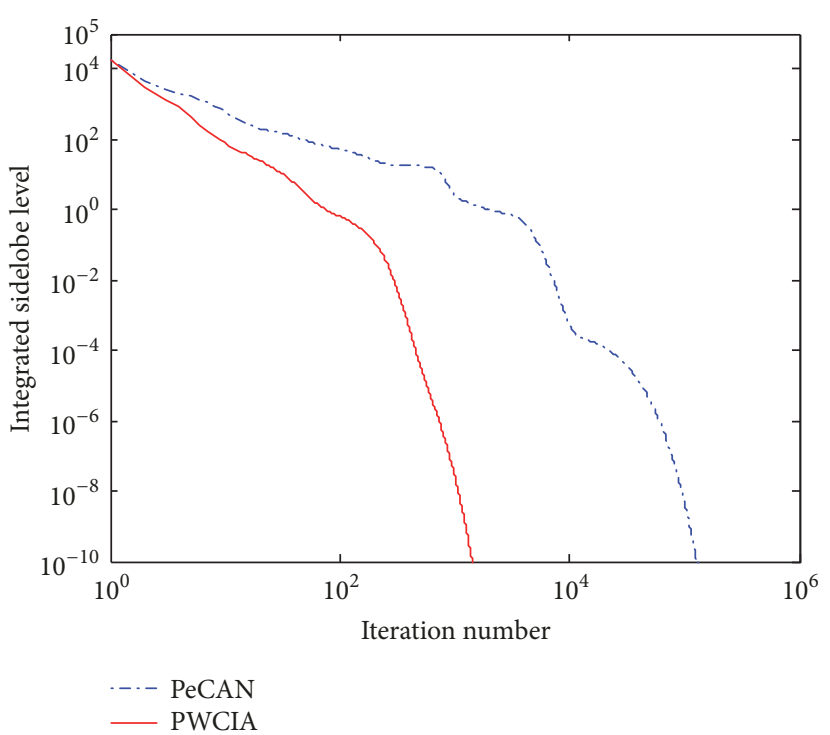

(a)

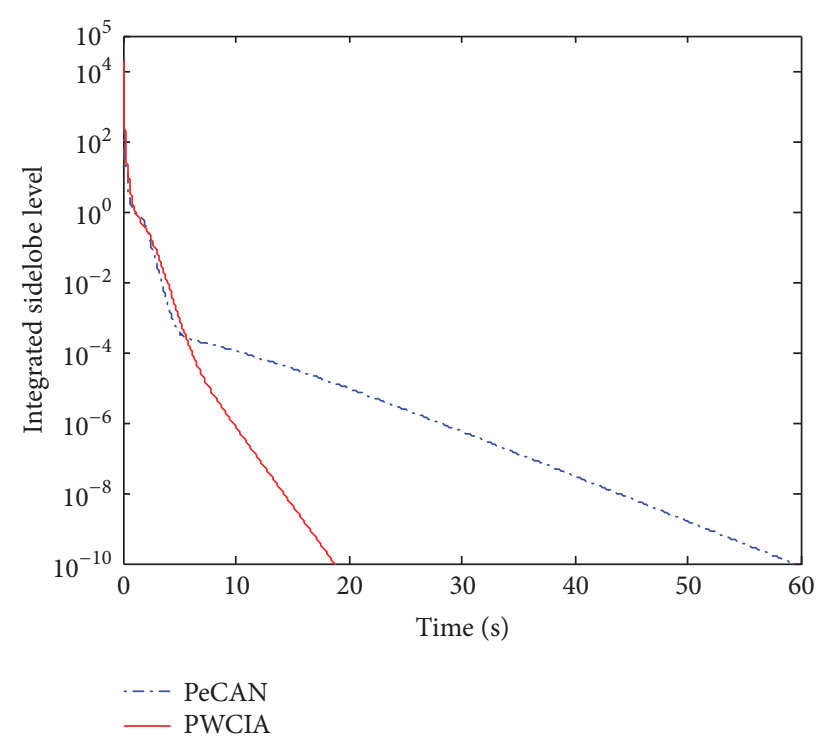

(b)

FIGURE 3: Integrated sidelobe level versus iteration number and computational time for $N=100$ : (a) iteration number; (b) time.

than that of the PeCAN at each iteration. Therefore, as the stopping criterion becomes more and more strict, the computational time cost by PWCIA is less than that of the PeCAN. This is reasonable probably because the PWCIA decomposes the $\mathrm{N}$-dimensional optimization problem into $N$ one-dimensional optimization problems and searches for the optimal solution one by one directly at each iteration, whereas the PeCAN requires only two FFT operations to solve an "almost equivalent" $N$-dimensional optimization problem at each iteration. However, it is worth noting that when the sequence length becomes long, such as $N=10^{4}$ or longer, the computational burden of PWCIA will be much more than the PeCAN.

4.1.2. Finite Phase Scenario. In this subsection, we assess the capability of the PWCIA for the finite phase scenario. Since the PeCAN has not proposed a specific method for finite phase case, in this experiment, we first use the PeCAN to generate the optimal sequence with continuous phase and then quantify the generated sequence to obtain the optimal sequence with finite phase.

Similar to the continuous phase case, we first compare the merit factor (MF) of the output sequences generated by two different algorithms. In this experiment, for both algorithms the stopping criterion was set as $\left\|\mathbf{x}^{(i)}-\mathbf{x}^{(i-1)}\right\|_{2}<10^{-6}$, and the other settings were the same in the case of continuous phase. We considered the following numbers of finite phase: $q=8,32,128$ for each of sequence lengths $(N=25,50,100$, $200,400,800$, and 1000). Furthermore, in order to illustrate the $q$ 's impact on the merit factor, the initial sequences are set to be the same. The average merit factor (MF) of the output sequences under different conditions is shown in Figure 4. From Figure 4, it can be observed that all the merit factors of output sequences with finite phase are smaller than the

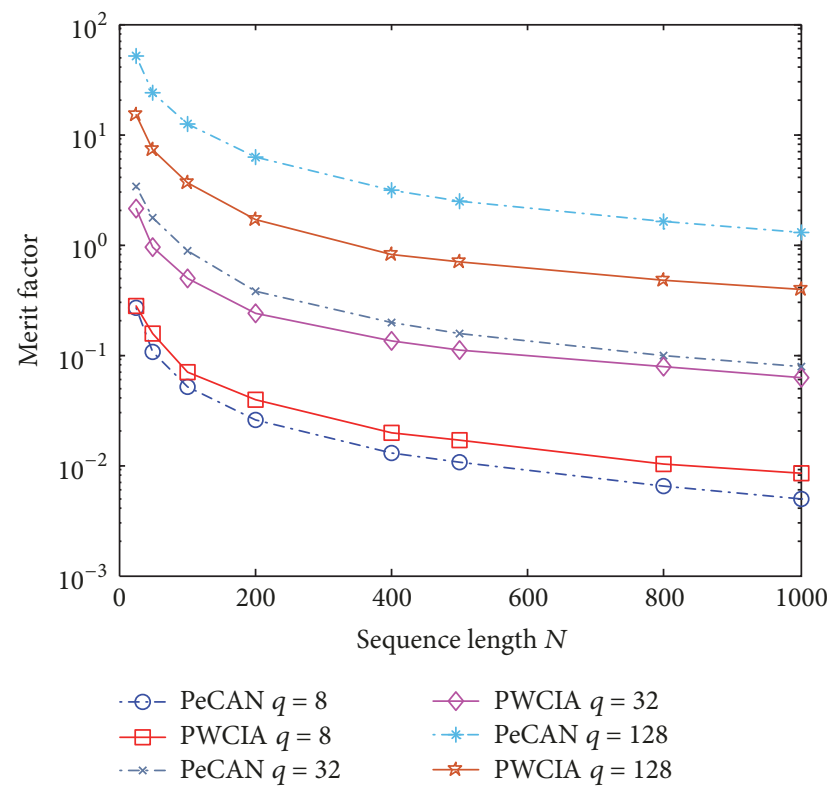

FIGURE 4: Merit factor (MF) versus sequence length (for finite phase). Each curve is averaged over 100 random trials.

continuous phase case and become larger as the number of finite phases $(q)$ increases for both algorithms. For small $q$, the merit factor of PWCIA sequence is larger than the PeCAN sequence, but for large $q$ the opposite is true. This is due to the fact that when the value of $q$ reaches a certain degree, the generated optimal sequence with finite phase will approach the optimal sequence with continuous phase. For PeCAN, the optimal sequence of finite phase is obtained by quantifying the continuous phase optimal sequence generated by PeCAN, 
TABLE 1: The average computational time (in seconds) of PWCIA and PeCAN over 100 random trials.

\begin{tabular}{lcc}
\hline$(N, q)$ & PWCIA & PeCAN \\
\hline$N=25, q=8$ & 0.0127 & \\
$N=25, q=32$ & 0.0179 & 0.3398 \\
$N=25, q=128$ & 0.0367 & \\
$N=100, q=8$ & 0.0667 & \\
$N=100, q=32$ & 0.1216 & 10.8837 \\
$N=100, q=128$ & 0.3061 & \\
$N=1000, q=8$ & 5.8096 & 956.3168 \\
$N=1000, q=32$ & 13.0924 & \\
$N=1000, q=128$ & 27.9178 &
\end{tabular}

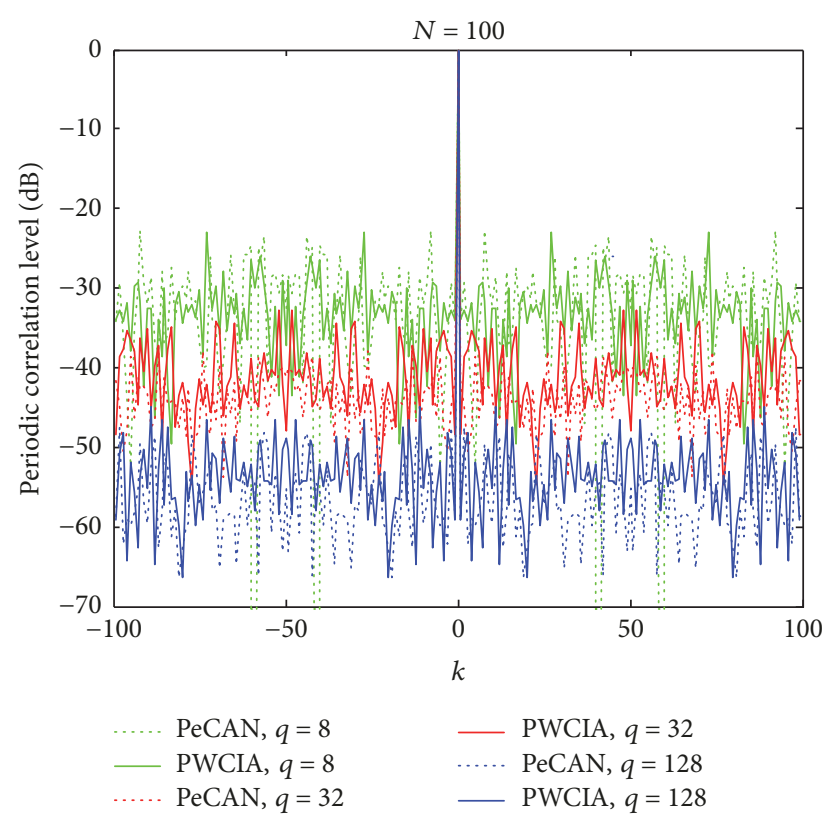

Figure 5: Periodic correlation levels of sequences generated by PeCAN and PWCIAs for $q=8,32,128, N=100$.

while for the PWCIA the optimal sequence of finite phase is obtained directly. Therefore, as the value of $q$ increases to a certain degree, the MF of the sequence generated by PeCAN will be larger than that of PWCIA.

Since the PeCAN does not have an alternative algorithm specifically for designing optimal sequence with finite phase, it is not meaningful to show the relationship between ISL and iteration numbers directly. Therefore, we just present the average computational time of two algorithms under different conditions in Table 1. Results show that for the finite phase case the PWCIA converges very fast compared with the PeCAN. Furthermore, as both $q$ and $N$ increase, the computational burden of PWCIA becomes larger and larger. The periodic correlation levels of finite phase optimal sequences of length $N=100$ generated separately by the two algorithms for $q=8,32,128$ are shown in Figure 5 .
4.2. WISL Metric. Compared with the other existing algorithms, the proposed PWCIA can generate sequences with not only low ISL but also low weighted ISL. In this subsection, we concentrated our attention on the PWCIA performance for WISL metric. Specifically, we consider the length of the designed sequence $N=100$, and all the sequences are initiated by the same unit energy sequence with random phases.

4.2.1. Continuous Phase Scenario. In the first experiment, we suppose that the main interference is from nearby, so we are interested in suppressing the periodic correlations $r(1), \ldots, r(15)$. To achieve this, we set the correlation coefficient weights $\left\{w_{k}\right\}_{k=1}^{N-1}$ as follows:

$$
w_{k}= \begin{cases}1, & k \in\{1, \ldots, 15\} \\ 0, & k \in\{16, \ldots, 99\}\end{cases}
$$

such that only the autocorrelations at the required lags will be minimized. Then, in the second experiment, we suppose that the main interference is from the middle-distance zone, and the aim is suppressing the periodic correlations $r(16), \ldots, r(35)$. Thus, we set correlation coefficient weights $\left\{w_{k}\right\}_{k=1}^{N-1}$ as follows:

$$
w_{k}= \begin{cases}1, & k \in\{16, \ldots, 35\} \\ 0, & \text { otherwise }\end{cases}
$$

Additionally, in order to reveal the convergence properties of PWCIA for WISL metric, the iteration number in the two experiments is set to 100 . The periodic correlation levels of the two designed sequences are shown in Figure 6. We can see in Figure 6 that the periodic correlation sidelobes of PWCIA sequences are suppressed to practically zero (about $-330 \mathrm{~dB}$ ) at the interesting lags. The WISL versus the iteration number and the corresponding computational time are shown in Figure 7. Results show that the proposed PWCIA converges very quickly compared with the ISL metric case. For the ISL metric case, the PWCIA needs over 1000 iterations and about 20 seconds to make the ISL decrease below $10^{-10}$, while for the WISL metric case the PWCIA takes iteration number of only 30 and about 0.4 seconds to drive the WISL to $10^{-28}$. Besides, if fewer periodic correlation lags are taken into account, the convergence speed of PWCIA becomes faster and the WISL of generated sequence becomes lower. The reason is that more and more degrees of freedom of the sequence $\{x(n)\}_{n=1}^{N}$ can be used to control $|r(k)|$ as the number of concerned periodic correlation lags decreases.

It is worth pointing out that the periodic correlation function $r(k)$ of $\{x(n)\}_{n=1}^{N}$ is also a periodic function of period $N$, which means $r(k)=r(k+N)$. With the help of formula (1), the relationship expression can be rewritten as

$$
\left|r\left(k-\frac{N}{2}\right)\right|=\left|r\left(k+\frac{N}{2}\right)\right|=\left|r\left(\frac{N}{2}-k\right)\right| .
$$




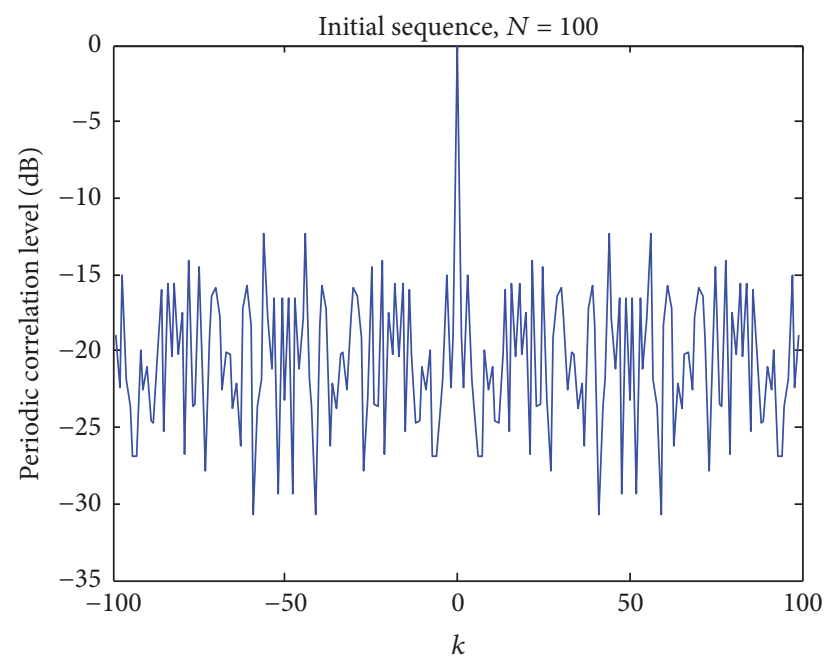

(a)

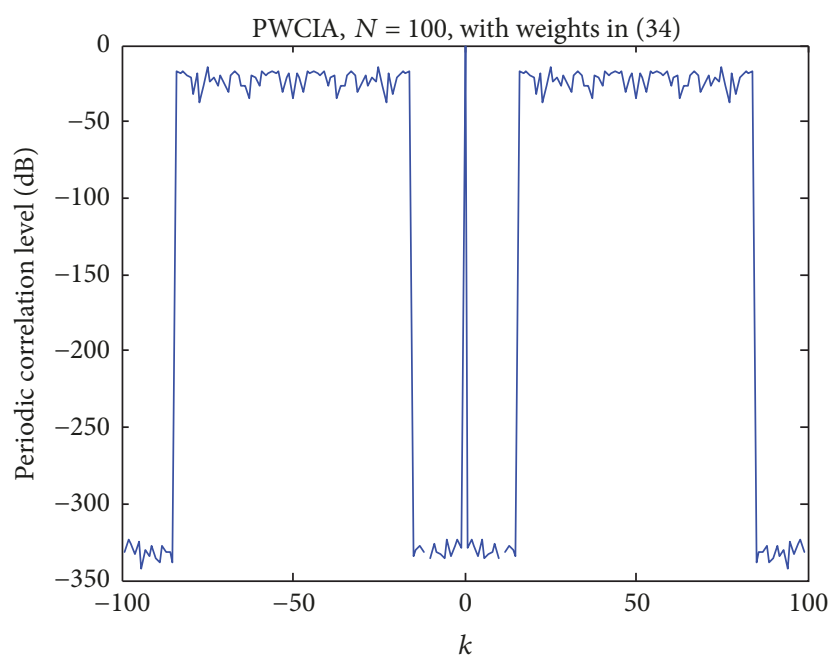

(b)

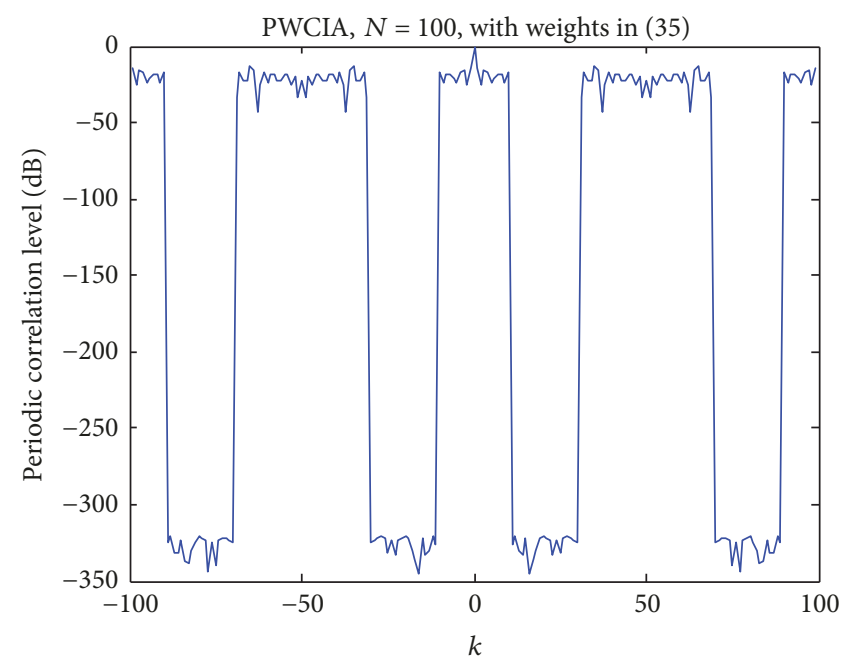

(c)

FIgURE 6: Periodic correlation level of the initial sequence and the PWCIA sequences of lengths $N=100$ designed under the WISL metric with weights in (34) and (35). (a) Initial sequence; (b) PWCIA sequence with weights in (34); (c) PWCIA sequence with weights in (35).

From (36), we can see that the degrees of freedom of the periodic correlation function are only $N / 2$ for sequence $\{x(n)\}_{n=1}^{N}$, which is the reason why criteria $C_{1}$ and $C_{2}$ are identical in [11].

4.2.2. Finite Phase Scenario. In this subsection, we use the PWCIA to design sequences with the same correlation coefficient weights in (34) and (35) for the finite phase case and consider the following numbers of finite phases: $q=$ $8,32,128$. The iteration number in the two experiments is set to 100 , which is the same as the continuous phase case. The periodic correlation levels of sequences generated by PWCIA for $q=8,32,128$ and weights in (34) and (35) are shown in Figure 8. The WISL versus the iteration number and the corresponding computational time are shown in Figure 9.
Results show that the periodic correlation level of output sequence with finite phase is worse than that with continuous phase at interesting lags. Furthermore, the smaller $q$ is, the worse the suppression effect is. Besides, the convergence speed of PWCIA for finite phase case is very fast. The PWCIA takes less than 10 iterations and less than 0.05 seconds to converge to a stationary point.

\section{Conclusions}

We have presented an efficient cyclic algorithm named PWCIA, which can be used to design unimodular sequences that have good periodic autocorrelation properties. The PWCIA decomposes the $N$-dimensional optimization problem into $N$ one-dimensional optimization problems and 


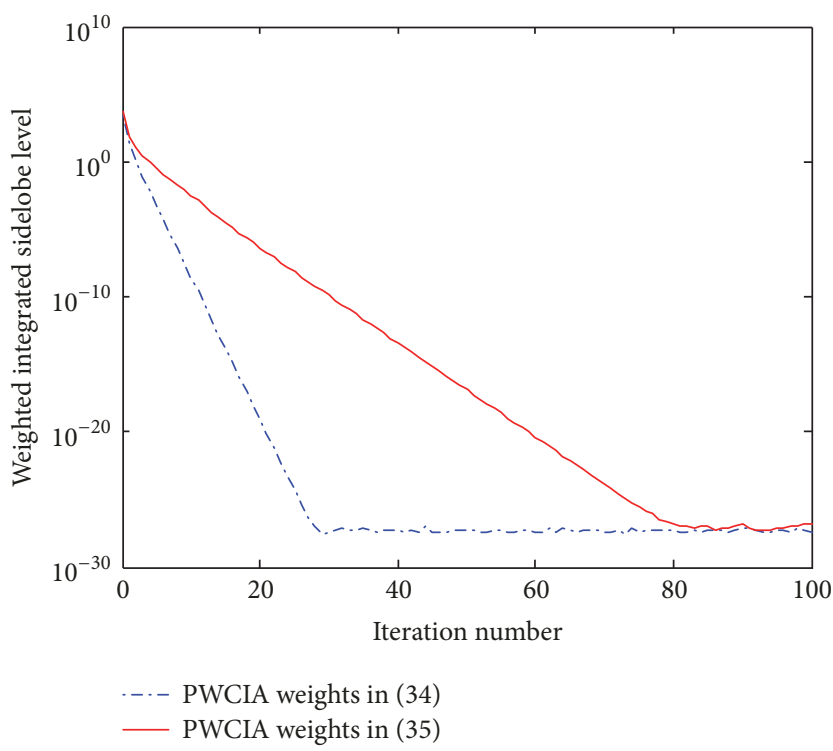

(a)

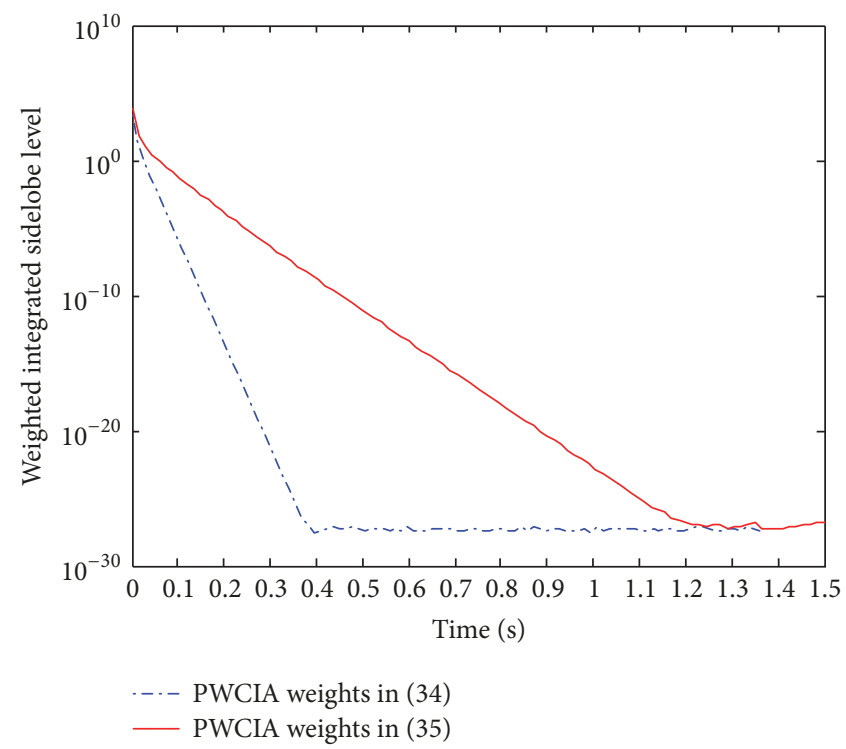

(b)

FIGURE 7: Weighted integrated sidelobe level versus iteration number and computational time (PWCIA, for continuous phase case). (a) Iteration number; (b) time.

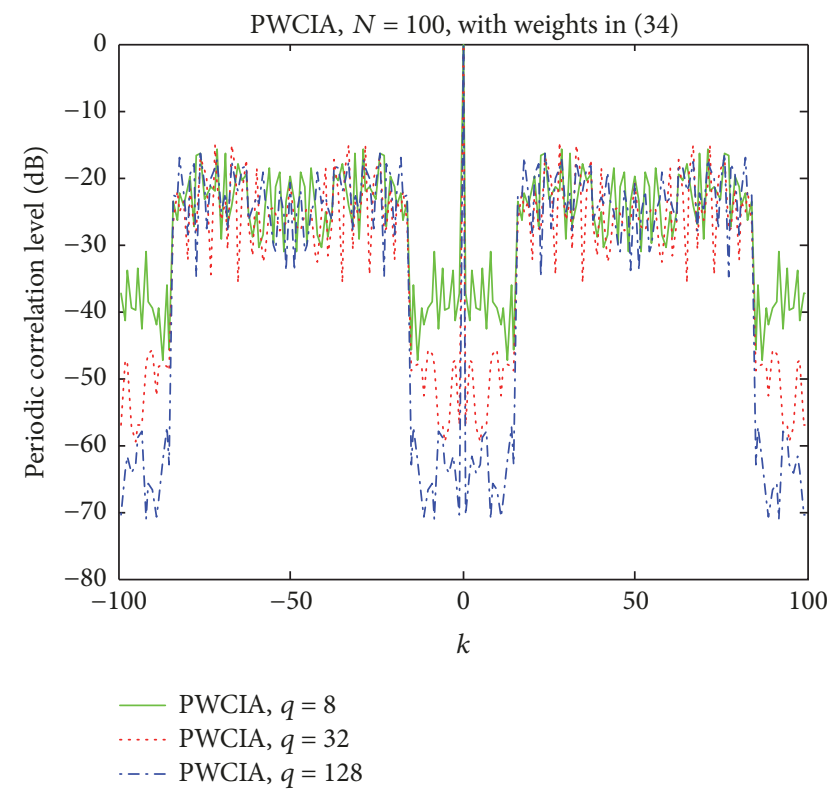

(a)

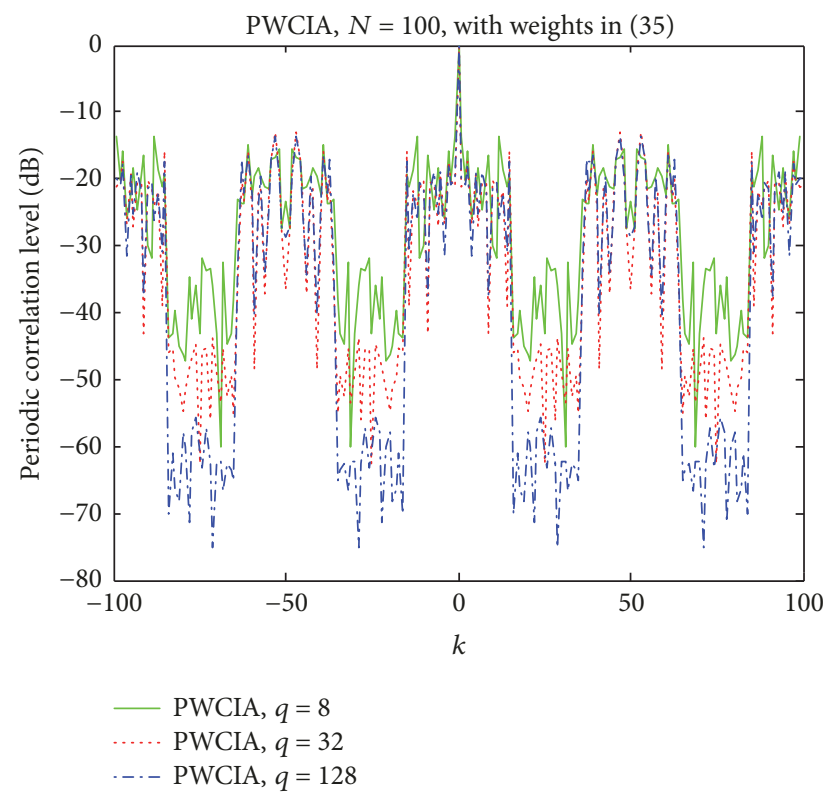

(b)

Figure 8: Periodic correlation levels of the PWCIA sequences for $q=8,32,128$ and $N=100$ designed under the WISL metric with weights in (34) and (35). (a) PWCIA sequence with weights in (34); (b) PWCIA sequence with weights in (35).

searches for the optimal solution directly at each iteration, which makes the convergence rate of this algorithm very fast. A number of numerical results show that the proposed PWCIA can not only deal with the periodic ISL metric but also firstly deal with the periodic WISL metric for both continuous and finite phase case. Additionally, the PWCIA shares a faster convergence speed and lower periodic correlation level compared with the PeCAN for both the continuous and finite phase case.

\section{Conflicts of Interest}

The authors declare that there are no conflicts of interest regarding the publication of this paper. 


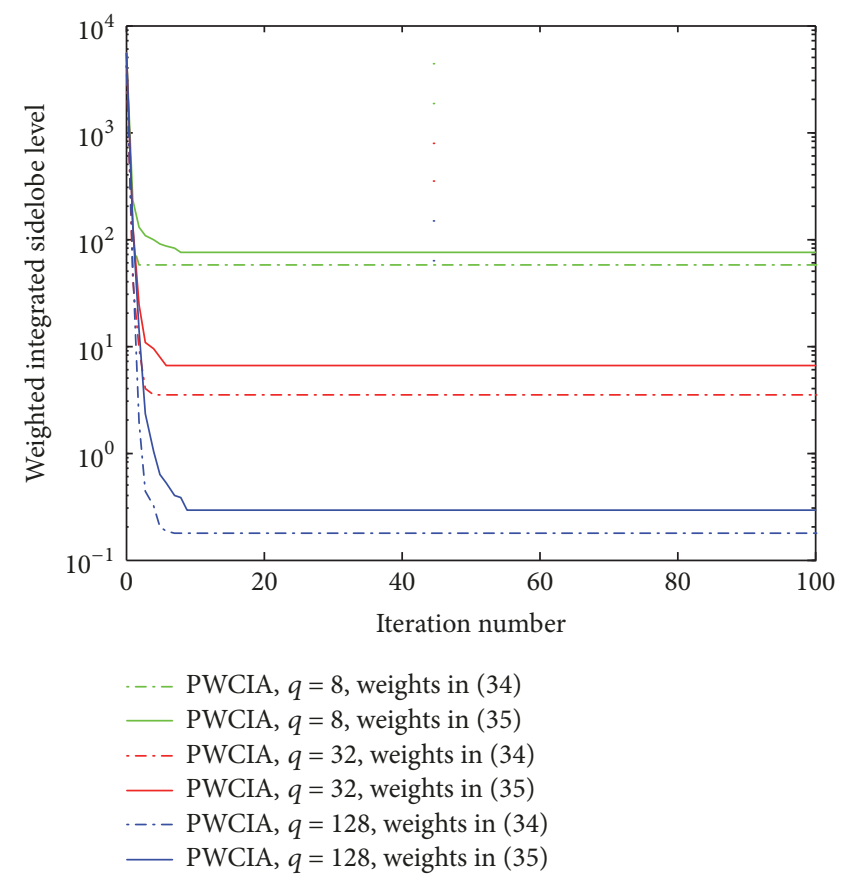

(a)

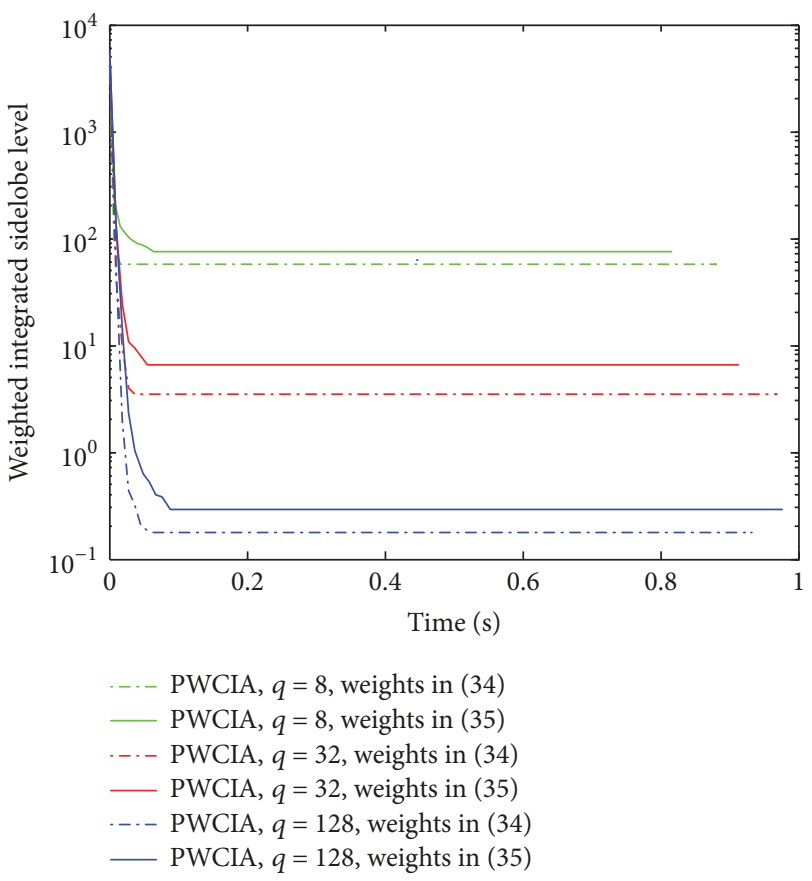

(b)

FIGURE 9: Weighted integrated sidelobe level versus iteration number and computational time (PWCIA, for finite phase case). (a) Iteration number; (b) time.

\section{Acknowledgments}

This work was supported by the National Natural Science Foundation of China under Grant no. 61673066 and the State Key Program of Basic Research of China under Grant no. 613196.

\section{References}

[1] S. D. Blunt and E. L. Mokole, "Overview of radar waveform diversity," IEEE Aerospace and Electronic Systems Magazine, vol. 31, no. 11, pp. 2-40, 2016.

[2] X. Gong, H. Meng, Y. Wei, and X. Wang, "Phase-modulated waveform design for extended target detection in the presence of clutter," Sensors, vol. 11, no. 7, pp. 7162-7177, 2011.

[3] S. W. Golomb and G. Gong, Signal Design for Good Correlation: for Wireless Communication, Ctyptography, and Rader, Cambridge University Press, Cambridge, UK, 2005.

[4] H. He, Waveform design for active sensing systems - a computational approach [Ph.D. thesis], University of Florida, 2011.

[5] J. Li, J. R. Guerci, and L. Xu, "Signal waveform's optimal-underrestriction design for active sensing," IEEE Signal Processing Letters, vol. 13, no. 9, pp. 565-568, 2006.

[6] W. Yue, Y. Zhang, Y. Liu, and J. Xie, "Radar constant-modulus waveform design with prior information of the extended target and clutter," Sensors, vol. 16, no. 6, article no. 889, 2016.

[7] E. Adams, M. Gouda, and P. Hill, "Detection and characterisation of DS/SS signals using higher-order correlation," in Proceedings of the ISSSTA'95 4th International Symposium on Spread Spectrum Techniques and Applications, pp. 27-31, Mainz, Germany, September 1996.
[8] H. Deng, "Discrete frequency-coding waveform design for netted radar systems," IEEE Signal Processing Letters, vol. 11, no. 2, pp. 179-182, 2004.

[9] P. Borwein and R. Ferguson, "Polyphase sequences with low autocorrelation," IEEE Transactions on Information Theory, vol. 51, no. 4, pp. 1564-1567, 2005.

[10] C. J. Nunn and G. E. Coxson, "Polyphase pulse compression codes with optimal peak and integrated sidelobes," IEEE Transactions on Aerospace and Electronic Systems, vol. 45, no. 2, pp. 775-781, 2009.

[11] P. Stoica, H. He, and J. Li, "New algorithms for designing unimodular sequences with good correlation properties," IEEE Transactions on Signal Processing, vol. 57, no. 4, pp. 1415-1425, 2009.

[12] P. Stoica, H. He, and J. Li, "On designing sequences with impulse-like periodic correlation," IEEE Signal Processing Letters, vol. 16, no. 8, pp. 703-706, 2009.

[13] H. He, P. Stoica, and J. Li, "Designing unimodular sequence sets with good correlations - Including an application to MIMO radar," IEEE Transactions on Signal Processing, vol. 57, no. 11, pp. 4391-4405, 2009.

[14] M. Soltanalian, M. M. Naghsh, and P. Stoica, "A fast algorithm for designing complementary sets of sequences," Signal Processing, vol. 93, no. 7, pp. 2096-2102, 2013.

[15] R. Manda and P. R. Kumar, "Performance comparison of unimodular sequence using CA, CAN, WeCAN algorithms," in Proceedings of the 2016 International Conference on Electrical, Electronics, and Optimization Techniques, ICEEOT 2016, pp. 1670-1676, Chennai, India, March 2016.

[16] J. Song, P. Babu, and D. P. Palomar, "Optimization methods for designing sequences with low autocorrelation sidelobes," IEEE 
Transactions on Signal Processing, vol. 63, no. 15, pp. 3998-4009, 2015.

[17] J. Song, P. Babu, and D. P. Palomar, "Sequence design to minimize the weighted integrated and peak sidelobe levels," IEEE Transactions on Signal Processing, vol. 64, no. 8, pp. 2051-2064, 2016.

[18] J. Song, P. Babu, and D. P. Palomar, "Sequence Set Design with Good Correlation Properties Via Majorization-Minimization," IEEE Transactions on Signal Processing, vol. 64, no. 11, pp. 28662879, 2016.

[19] M. Soltanalian and P. Stoica, "Computational design of sequences with good correlation properties," IEEE Transactions on Signal Processing, vol. 60, no. 5, pp. 2180-2193, 2012.

[20] G. Cui, X. Yu, M. Piezzo, and L. Kong, "Constant modulus sequence set design with good correlation properties," Signal Processing, vol. 139, pp. 75-85, 2017.

[21] L. Tang, Y. Zhu, and Q. Fu, "Designingwaveform sets with good correlation and stopband properties for MIMO radar via the gradient-based method," Sensors, vol. 17, no. 5, article no. 999, 2017.

[22] I. A. Arriaga-Trejo, A. G. Orozco-Lugo, and J. Flores-Troncoso, "Design of Unimodular Sequences with Good Autocorrelation and Good Complementary Autocorrelation Properties," IEEE Signal Processing Letters, vol. 24, no. 8, pp. 1153-1157, 2017.

[23] N. Levanon, "Noncoherent radar pulse compression based on complementary sequences," IEEE Transactions on Aerospace and Electronic Systems, vol. 45, no. 2, pp. 742-747, 2009.

[24] H. C. Zhao, Fundamentals and Methodology of Radio Fuze, National Defense Industry Press, Beijing, China, 2012.

[25] G. Xiong, X.-N. Yang, and H.-C. Zhao, "Inter-pulse pseudorandom binary-phase code and linear frequency modulation combined detecting system," Binggong Xuebao/Acta Armamentarii, vol. 28, no. 4, pp. 425-430, 2007. 


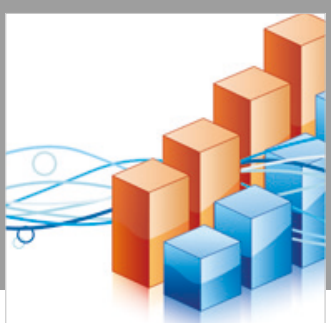

Advances in

Operations Research

\section{-n-m}
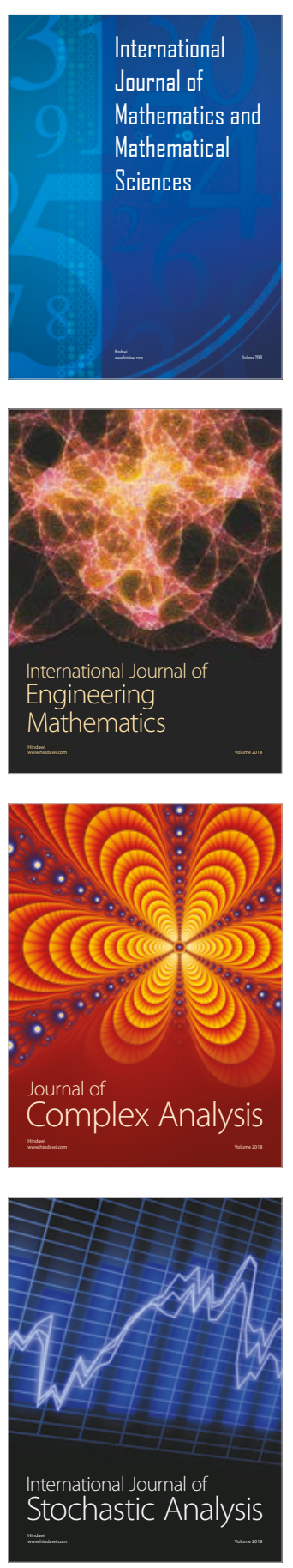
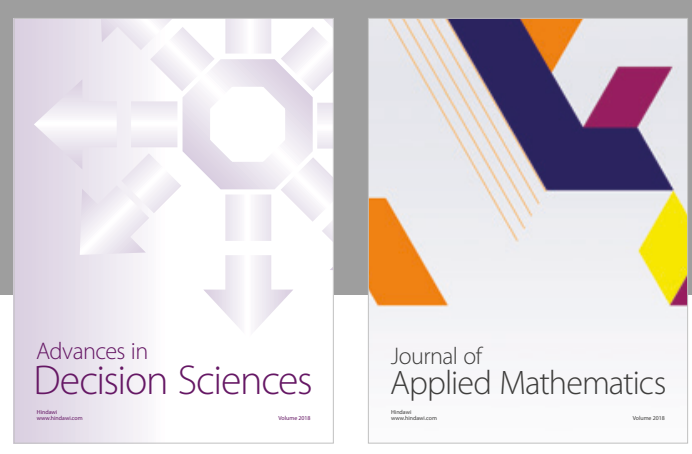

Journal of

Applied Mathematics
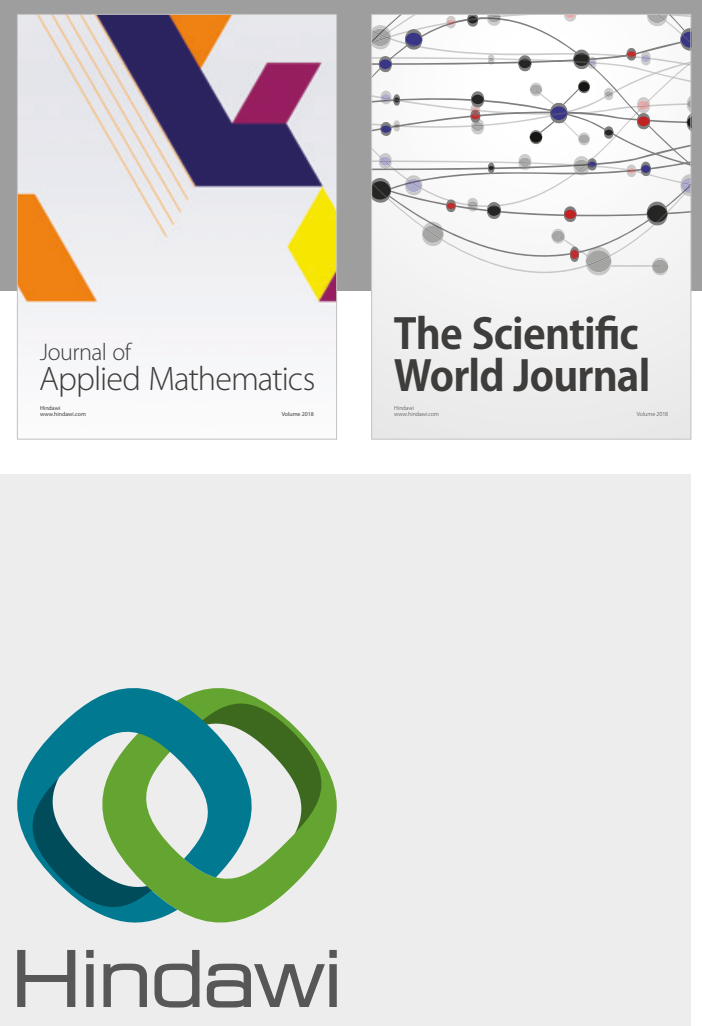

Submit your manuscripts at

www.hindawi.com

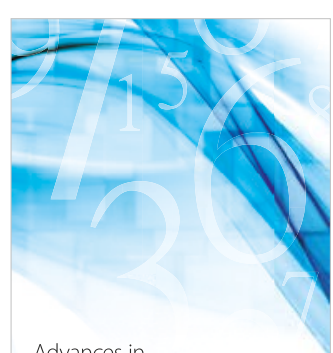

Advances in
Numerical Analysis
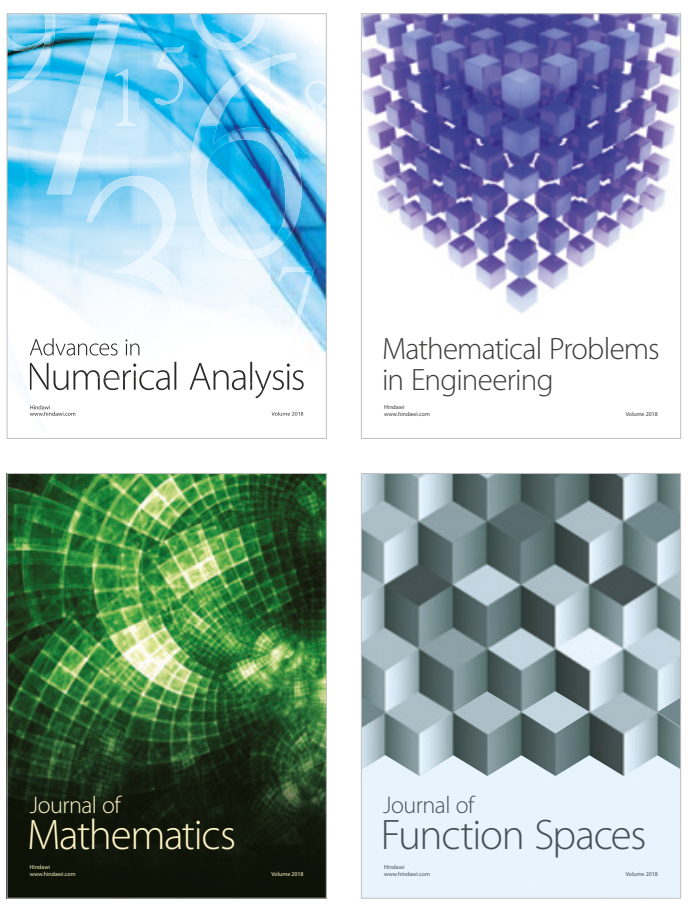

Mathematical Problems in Engineering

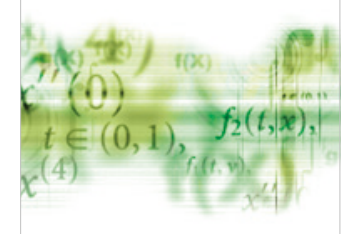

International Journal of

Differential Equations

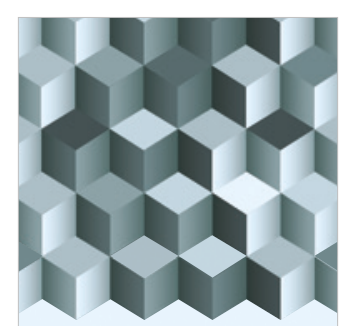

Journal of

Function Spaces

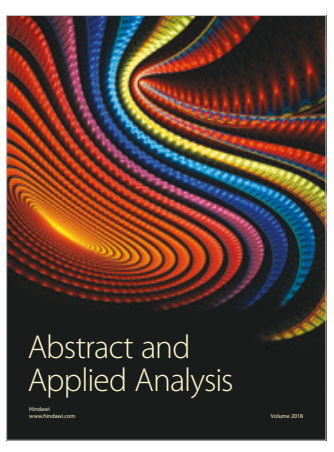

The Scientific

World Journal

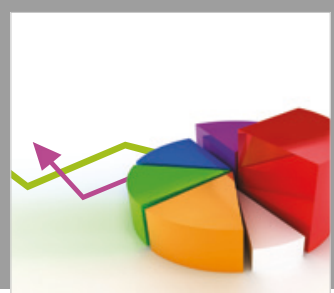

Journal of

Probability and Statistics
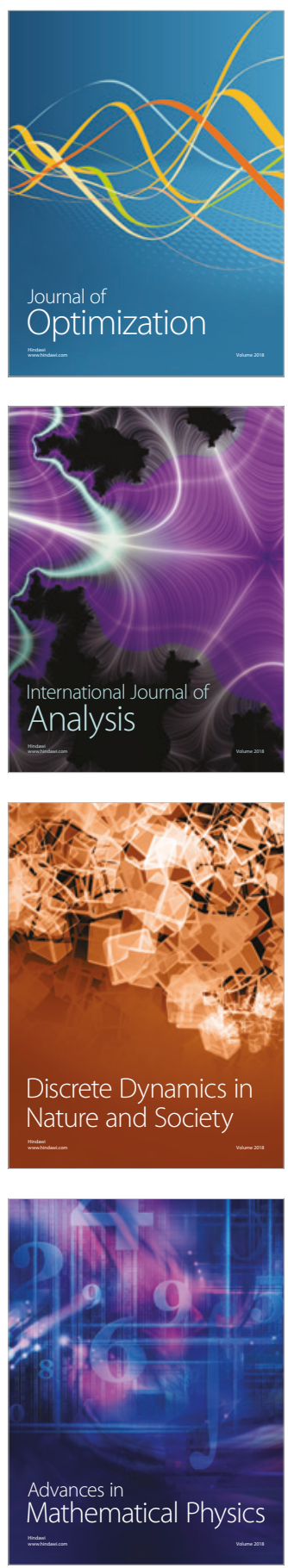